MATHEMATICS OF COMPUTATION

Volume 75, Number 255, July 2006, Pages 1033-1042

S $0025-5718(06) 01829-1$

Article electronically published on March 13, 2006

\title{
ERROR REDUCTION AND CONVERGENCE FOR AN ADAPTIVE MIXED FINITE ELEMENT METHOD
}

\author{
CARSTEN CARSTENSEN AND R. H. W. HOPPE
}

\begin{abstract}
An adaptive mixed finite element method (AMFEM) is designed to guarantee an error reduction, also known as saturation property: after each refinement step, the error for the fine mesh is strictly smaller than the error for the coarse mesh up to oscillation terms. This error reduction property is established here for the Raviart-Thomas finite element method with a reduction factor $\rho<1$ uniformly for the $L^{2}$ norm of the flux errors. Our result allows for linear convergence of a proper adaptive mixed finite element algorithm with respect to the number of refinement levels. The adaptive algorithm surprisingly does not require any particular mesh design, unlike the conforming finite element method. The new arguments are a discrete local efficiency and a quasi-orthogonality estimate. The proof does not rely on duality or on regularity.
\end{abstract}

\section{INTRODUCTION}

An adaptive finite element method consists of successive loops of the following sequence:

$$
\mathrm{SOLVE} \rightarrow \text { ESTIMATE } \rightarrow \text { MARK } \rightarrow \text { REFINE. }
$$

The a posteriori error control in the step ESTIMATE has been developed over the last decades (cf. 1, 3, 6, 12, 17 and the references therein). The convergence analysis of the full algorithm (1.1), however, is restricted to the conforming finite element method [15, 16.

This paper investigates convergence properties of such a loop for the mixed finite element method (MFEM) in a 2D model Poisson problem

$$
f+\Delta u=0 \text { in } \Omega \text { and } u=0 \text { on } \partial \Omega .
$$

Given a (coarse) mesh $\mathcal{T}_{H}$, a shape-regular triangulation of $\Omega$ into triangles, $p_{H}$ and $u_{H}$ approximate the exact flux $p:=\nabla u \in H(\operatorname{div}, \Omega)$ and the exact displacement field $u \in H_{0}^{1}(\Omega)$ of (1.2). In step SOLVE one computes $\left(p_{H}, u_{H}\right) \in$ $R T_{0}\left(\mathcal{T}_{H}\right) \times P_{0}\left(\mathcal{T}_{H}\right)$ that satisfies the discrete problem $\left[(\bullet, \bullet)_{L^{2}}\right.$ abbreviates the $L^{2}$ scalar product]

$$
\begin{array}{cl}
\left(p_{H}, q_{H}\right)_{L^{2}(\Omega)}+\left(u_{H}, \operatorname{div} q_{H}\right)_{L^{2}(\Omega)}=0 & \text { for all } q_{H} \in R T_{0}\left(\mathcal{T}_{H}\right), \\
\left(\operatorname{div} p_{H}, v_{H}\right)_{L^{2}(\Omega)}=-\left(f, v_{H}\right)_{L^{2}(\Omega)} & \text { for all } v_{H} \in P_{0}\left(\mathcal{T}_{H}\right) .
\end{array}
$$

Details on the lowest-order Raviart-Thomas finite element space $R T_{0}\left(\mathcal{T}_{H}\right)$ [ can be found below in Section 2, $P_{0}\left(\mathcal{T}_{H}\right)$ denotes the piecewise constants. MATLAB

Received by the editor April 11, 2004.

2000 Mathematics Subject Classification. Primary 65N30, 65N50. 


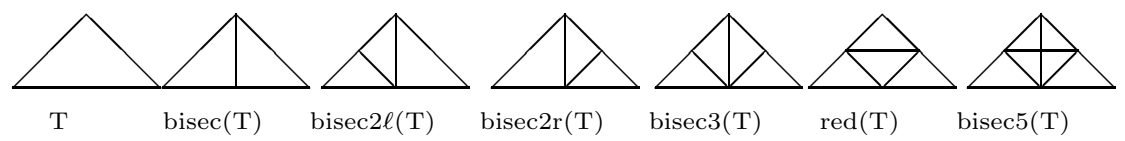

Figure 1. Possible refinements of one triangle $T$ in the step REFINE.

implementations and documentations of the step SOLVE are provided in [5]. In this paper, for the ease of discussion, the step ESTIMATE is the postprocessing to compute the residual-based explicit error estimator [2, 9, 18 ]

$$
\eta_{H}:=\left(\sum_{E \in \mathcal{E}_{H}} \eta_{E}^{2}\right)^{1 / 2} \quad \text { with } \eta_{E}^{2}:=h_{E}\left\|\left[p_{H}\right]_{E}\right\|_{L^{2}(E)}^{2} .
$$

Here and throughout, $\left[p_{H}\right]$ denotes the jump $\left[p_{H}\right]:=\left.p_{H}\right|_{T_{+}}-\left.p_{H}\right|_{T_{-}}$of the discrete flux over an interior edge $E:=T_{+} \cap T_{-}$of length $h_{E}:=\operatorname{diam}(E)$ shared by the two neighboring (closed) triangles $T_{ \pm} \in \mathcal{T}_{H}$. Furthermore let $f_{\omega_{E}}:=\left|\omega_{E}\right|^{-1} \int_{\omega_{E}} f(x) d x$ denote the integral mean of $f$ over the patch $\omega_{E}:=\operatorname{int}\left(T_{+} \cup T_{-}\right)$of area $\left|\omega_{E}\right|=$ $\left|T_{+}\right|+\left|T_{-}\right|$and let $\mathcal{E}_{H}$ denote the set of all interior edges in $\mathcal{T}_{H}$.

The bulk criterion in the step MARK was introduced and analyzed in [7, 11, 15. for displacement-based AFEMs. Here, it leads to a selection of a subset $\mathcal{M}$ of edges $\mathcal{E}_{H}$ such that

$$
\theta \eta_{H}^{2} \leq \sum_{E \in \mathcal{M}} \eta_{E}^{2}
$$

for some universal constant $0<\theta<1$. It came much as a surprise to the authors that the step REFINE does not need any further specification or restriction. It suffices when the output of REFINE satisfies that, for each marked edge $E \in \mathcal{M}$, its midpoint $\operatorname{mid}(E)$ is a new node in the new triangulation $\mathcal{T}_{h}$.

Typical refinements of one triangle $T \in \mathcal{T}_{H}$ are displayed in Figure 1 .

We further set $h_{T}:=\operatorname{diam}(T)$ and refer to $\left\|H f_{H}\right\|_{L^{2}(\Omega)}$ as the first-order term given by

$$
\left\|H f_{H}\right\|_{L^{2}(\Omega)}:=\left(\sum_{T \in \mathcal{T}_{H}} h_{T}^{2}|T|^{-1}\left|\int_{T} f(x) d x\right|^{2}\right)^{1 / 2},
$$

while the data oscillations read

$$
\operatorname{osc}_{H}:=\left(\sum_{E \in \mathcal{E}_{H}} h_{E}^{2}\left\|f-f_{\omega_{E}}\right\|_{L^{2}\left(\omega_{E}\right)}^{2}\right)^{1 / 2}
$$

It is the milestone of this paper to prove the following error reduction property (1.8).

Theorem 1.1 (Error reduction property). Let $p_{h}$ and $p_{H}$ be the MFEM flux approximations to $p$ with respect to $\mathcal{T}_{h}$ and $\mathcal{T}_{H}$. Then, there exist positive constants $\rho<1$ and $C$ depending only on $\theta$ and on the shape regularity of $\mathcal{T}_{h}$ and $\mathcal{T}_{H}$ such that

$$
\left\|p-p_{h}\right\|_{L^{2}(\Omega)}^{2} \leq \rho\left\|p-p_{H}\right\|_{L^{2}(\Omega)}^{2}+C\left(\left\|H f_{H}\right\|_{L^{2}(\Omega)}+\operatorname{osc}_{H}\right) \operatorname{osc}_{H} .
$$

The remaining part of this paper is organized as follows. Section 2 discusses several aspects of AMFEM as well as particularities and generalizations of our analysis. 
Section 3 presents the necessary details on the notation. The key ingredients of the proof are the strict discrete local efficiency, the quasi-orthogonality, and an estimate for the fluxes, of Section 4 and [5. The proof of the error reduction property (1.8) concludes the paper in Section 6 ,

\section{Comments}

Some remarks are given before the subsequent sections are devoted to the technical details of the proof of Theorem 1.1 .

2.1. Data oscillations. For $f \in H^{1}(\Omega)$, we note that the data oscillation (1.7) is of quadratic order and so of higher order when compared to the first-order errors $\left\|p-p_{H}\right\|_{H(\text { div })}$ or $\left\|u-u_{H}\right\|_{L^{2}(\Omega)}$ or the first-order data term $\left\|H f_{H}\right\|_{L^{2}(\Omega)}$.

Hence, Theorem 1.1 asserts that the error on the fine mesh is bounded by a factor $\rho^{1 / 2}$ times the error on the coarse mesh plus higher-order terms.

We also point out that the oscillations (1.7) of $f$ are patch-oriented while those in the reliability and efficiency estimate of Theorem 3.2 below are element-oriented (and so possibly smaller than (1.7)).

It is an important property of the data oscillation that the mesh-sizes enter explicitly. Given $0<\vartheta<1$ and a coarse mesh $\mathcal{T}_{H}$, it is therefore easy to design a fine mesh $\mathcal{T}_{h}$ with oscillations osc $h \leq \vartheta \operatorname{osc}_{H}$, where $\operatorname{osc}_{h}$ and $\operatorname{osc}_{H}$ denote the data oscillation of the fine and coarse mesh, respectively. The same remark applies to $\left\|H f_{H}\right\|_{L^{2}(\Omega)}$.

2.2. A convergent AMFEM. In order to guarantee linear convergence in terms of the refinement levels, suppose that (1.1) is employed successively. At the refinement level $j$, there is an MFEM solution $p_{j}$ with error $e_{j}:=\left\|p-p_{j}\right\|_{L^{2}(\Omega)}$ with respect to a mesh $\mathcal{T}_{j}$ and a data oscillation $\operatorname{osc}_{j}$ such that (1.8) reads

$$
e_{j+1}^{2} \leq \rho e_{j}^{2}+C d_{j} \quad \text { for } j=0,1,2, \ldots,
$$

where $d_{j}$ abbreviates the data term $\left(\left\|H_{j} f_{H_{j}}\right\|_{L^{2}(\Omega)}+\operatorname{osc}_{j}\right) \operatorname{osc}_{j}$ with respect to $\mathcal{T}_{j}$. Moreover, suppose that MARK provides (1.5) plus (possibly) additional refinements to guarantee

$$
d_{j+1} \leq \varrho d_{j} \quad \text { for } j=0,1,2, \ldots
$$

with some universal constant $0<\varrho<1$ (this is always possible, as indicated at the end of the previous subsection).

Mathematical induction proves that (2.1)-(2.2) imply

$$
e_{j}^{2} \leq \rho^{j} e_{0}^{2}+C d_{0} \sum_{k=0}^{j-1} \rho^{k} \varrho^{j-1-k} \quad \text { and } \quad d_{j} \leq d_{0} \varrho^{j},
$$

and so R-linear convergence (with any reduction factor between $\max \{\rho, \varrho\}^{1 / 2}$ and 1)

$$
e_{j}^{2} \leq \rho^{j} e_{0}^{2}+C d_{0} j \max \{\rho, \varrho\}^{j-1} \quad \text { for } j=1,2, \ldots
$$


2.3. Numerical experiments. Numerical experiments throughout the literature are frequently based on the element-oriented maximum criterion in the step MARK, i.e., one marks an element $T$ if the estimator $\eta_{T}$ associated with $T$ satisfies Tol $\leq \eta_{T}$ and Tol is $\theta$ times the largest of such contributions. In the context of AMFEM, data oscillations have not been involved so far. We refer to [5] for algorithmic details and MATLAB routines and to [2, 10, 18, 13, for empirical examples.

It is the authors' overall impression that the AMFEM is very robust in changing algorithmic details in practice. The numerical experiments in [15, 16] with a realization of (2.1) - (2.2) from the previous subsection for conforming AFEM anticipate that the new algorithms perform as optimally as the frequently employed ones. But there is no mathematical justification for that.

2.4. Optimal complexity. The adaptive algorithm is linear convergent with respect to the number of refinement steps. This does not imply any control of the number of degrees of freedom. Based on additional coarsening steps, there exists an algorithm of optimal complexity for the conforming AFEM [7]. The authors anticipate that their results carry over to the present situation, because it is the universal coarsening step that yields the control of the degrees of freedom. Numerical wisdom, however, tells us that coarsening is not needed in practice, leaving an open gap between theory and practice.

2.5. Generalizations. The arguments below are illustrated by a simple $2 \mathrm{D}$ model example only, but they apply to more general boundary value problems as well. In the presence of Neumann boundary data or for nonconstant coefficients, the data oscillations apply to such terms as well. The arguments are not restricted to 2D; for instance, Lemma 3.1 also holds true in 3D [5].

The use of alternative refinement indicators [10, 18, is also possible, as long as they are globally reliable and locally controlled by the residual-based estimators.

2.6. Uzawa algorithms. The well-established Uzawa algorithm for the iterative solution of the mixed problem on the continuous level consists of two steps: a Poisson solve and an update formula. The substitute of the Poisson solve by some AFEM allows a perturbation of the convergence on the continuous level [4]. The advantage is that even unstable finite element schemes can be employed. The disadvantage is the possibly slow convergence of the Uzawa algorithm relative to multilevel solver [13].

\section{Notation AND PRELIMINARIES}

Throughout this paper suppose that $\mathcal{T}_{H}$ and $\mathcal{T}_{h}$ are two shape regular triangulations of the planar Lipschitz domain $\Omega$ with polygonal boundary $\partial \Omega$ into triangles, where $\mathcal{T}_{h}$ is some refinement of $\mathcal{T}_{H}$ such that the refinement $\left.T\right|_{\mathcal{T}_{h}}:=\left\{K \in \mathcal{T}_{h}: K \subseteq\right.$ $T$ \} of each element $T$ in $\mathcal{T}_{H}$ is depicted in Figure 1. Moreover, let $p_{H} \in R T_{0}\left(\mathcal{T}_{H}\right)$ denote the discrete MFEM solution on the coarse triangulation $\mathcal{T}_{H}$. A regular triangulation $\mathcal{T}$ in triangles, $d=2$, is a set of closed triangles $T$ of positive area $|T|$ such that any two distinct triangles $T_{1}$ and $T_{2}$ are either disjoint $T_{1} \cap T_{2}=\emptyset$ or share exactly one vertex $z, T_{1} \cap T_{2}=\{z\}$, or have one edge $E=T_{1} \cap T_{2}$ in common. The set of all edges is denoted by $\mathcal{E}$, and the set of nodes is denoted by $\mathcal{N}$. Each edge is associated to a length $h_{E}:=\operatorname{diam}(E)$ and a unit normal and unit tangential vector $\nu_{E}$ and $\tau_{E}$. The subindices $H$ and $h$ refer to the coarse and fine triangulation 
$\mathcal{T}_{H}$ and $\mathcal{T}_{h}$, respectively. The words mesh and triangulation are used as synonyms of each other.

The Raviart-Thomas MFEM space and the piecewise constant space read

$$
\begin{aligned}
R T_{0}\left(\mathcal{T}_{H}\right):= & \left\{q_{H} \in H(\operatorname{div}, \Omega): \forall T \in \mathcal{T}_{H} \exists a \in \mathbb{R}^{2} \exists b \in \mathbb{R} \forall x \in T,\right. \\
& \left.q_{H}(x)=a+b x\right\}, \\
P_{0}\left(\mathcal{T}_{H}\right):= & \left\{v_{H} \in L^{\infty}(\Omega): \forall T \in \mathcal{T}_{H} \exists a \in \mathbb{R} \forall x \in T, v_{H}(x)=a\right\} .
\end{aligned}
$$

(Analogous notation for $\mathcal{T}_{h}$ is not displayed.) The Crouzeix-Raviart FEM space on $\mathcal{T}_{H}$ reads

$$
\begin{gathered}
V_{H}^{N}:=\left\{v_{H} \in P_{1}\left(\mathcal{T}_{H}\right): v_{H} \text { continuous at } \operatorname{mid}(E) \text { for } E \in \mathcal{E}_{H},\right. \\
\text { and } \left.v_{H}(\operatorname{mid}(E))=0 \text { for } E \in \mathcal{E} \text { with } E \subseteq \partial \Omega\right\} .
\end{gathered}
$$

Since $V_{H}^{N} \not \subset H^{1}(\Omega)$, the distributional gradient of $v_{h} \in V_{H}^{N}$ is different from its elementwise gradient $D_{H} v_{H} \in P_{0}\left(\mathcal{T}_{H}\right)^{d}$.

Let $u_{H}^{N}$ denote the Crouzeix-Raviart FEM solution of

$$
\left(D_{H} u_{H}^{N}, D_{H} v_{H}^{N}\right)_{L^{2}(\Omega)}=\left(f_{H}, v_{H}^{N}\right)_{L^{2}(\Omega)} \quad \text { for all } v_{H}^{N} \in V_{H}^{N} .
$$

The discrete fluxes $p_{H}^{N}:=D_{H} u_{H}^{N}$ and $p_{H}$ from (1.3) are related.

Lemma 3.1 ([14,,$[5])$. Let $f_{T_{ \pm}}:=\int_{T_{ \pm}} f(x) d x /\left|T_{ \pm}\right|$and let $x_{T_{ \pm}}:=\operatorname{mid}\left(T_{ \pm}\right)$denote the barycenter of $T_{ \pm}$. Then there holds

$$
\left.p_{H}\right|_{T_{ \pm}}(x)=\left.D_{H} u_{H}^{N}\right|_{T_{ \pm}}-\frac{1}{2} f_{T_{ \pm}}\left(x-x_{T_{ \pm}}\right) \quad \text { for } x \in T_{ \pm} .
$$

In this context, $f_{H} \in P_{0}\left(\mathcal{T}_{H}\right)$ and $f_{h} \in P_{0}\left(\mathcal{T}_{h}\right)$ denote the piecewise integral means, e.g., $\left.f_{H}\right|_{T}:=f_{T}:=\int_{T} f(x) d x /|T|$ for $T \in \mathcal{T}_{H}$.

Theorem 3.2 (Reliability and efficiency [2, 9]). With (1.4) and (1.7), there holds

$$
\eta_{H} \lesssim\left\|p-p_{H}\right\|_{L^{2}(\Omega)} \lesssim \eta_{H}+\operatorname{osc}_{H}
$$

Here and throughout this paper, $A \lesssim B$ abbreviates $A \leq C B$ with a mesh-size independent, generic constant $C>0$. Finally, $A \approx B$ abbreviates $A \lesssim B \lesssim A$. The paper adopts standard notation for Lebesgue and Sobolev spaces and norms.

\section{Discrete local EFficiency}

This section provides the first of two main arguments for error reduction. Unlike for the conforming AFEM, there is no request for further restriction in REFINE.

Theorem 4.1 (Strict discrete local efficiency). Suppose that $E=\partial T_{+} \cap \partial T_{-} \in$ $\mathcal{E}_{H}$ is an edge in $\mathcal{T}_{H}$ (shared by the triangles $T_{+}, T_{-} \in \mathcal{T}_{H}$ ) and bisected in the refinement, i.e., $E=E_{1} \cup E_{2} \notin \mathcal{E}_{h}$ and $\operatorname{mid}(E)=E_{1} \cap E_{2} \in \mathcal{N}_{h}$ for two distinct $E_{1}, E_{2} \in \mathcal{E}_{h}$. Then there holds

$$
h_{E}^{1 / 2}\left\|\left[p_{H}\right]\right\|_{L^{2}(E)} \lesssim\left\|p_{h}-p_{H}\right\|_{L^{2}\left(\omega_{E}\right)}+h_{E}\left\|f-f_{\omega_{E}}\right\|_{L^{2}\left(\omega_{E}\right)} .
$$

The remaining part of this section is devoted to the proof of Theorem 4.1. Observe that $\left[p_{H}\right] \cdot \nu_{E}=0$ for the unit normal vector $\nu_{E} \perp E$ since $p_{H} \in H(\operatorname{div}, \Omega)$. Therefore, denoting by $\tau_{E} \perp \nu_{E}$ the tangential vector, the jump

$$
\left[p_{H}\right]:=\left(\left.p_{H}\right|_{T_{+}}-\left.p_{H}\right|_{T_{-}}\right) \quad \text { along } E=T_{+} \cap T_{-}
$$

(and formally $\left[p_{H}\right]:=0$ along $E \subset \partial \Omega$ ) satisfies

$$
\left\|\left[p_{H}\right]\right\|_{L^{2}(E)}=\left\|\left[p_{H}\right] \cdot \tau_{E}\right\|_{L^{2}(E)} .
$$


Taking into account that $\left[p_{H}\right] \cdot \tau_{E}$ is an affine function along the edge $E$, we have

$$
\left(\left[p_{H}\right] \cdot \tau_{E}\right)(x)=\alpha+\beta \cdot(x-\operatorname{mid}(E)) \text { for all } x \in E
$$

with fixed $\alpha \in \mathbb{R}$ and $\beta \in \mathbb{R}^{2}$.

Lemma 4.2. There holds

$$
h_{E}^{1 / 2}\|\alpha\|_{L^{2}(E)} \lesssim\left\|p_{h}-p_{H}\right\|_{L^{2}\left(\omega_{E}\right)} .
$$

Proof. Let $\varphi_{E}$ denote the nodal basis function in the conforming $P_{1}$ FEM space with respect to the node $\operatorname{mid}(E)$ and with respect to the fine mesh $\mathcal{T}_{h}$. Then, $q_{h}:=$ Curl $\varphi_{E}$ belongs to $P_{0}\left(\mathcal{T}_{h}\right) \cap H(\operatorname{div}, \Omega)$ with $\operatorname{div} q_{h} \equiv 0$. Since $\alpha=\int_{E}\left[p_{H}\right] \cdot \tau_{E} d s / h_{E}$, one deduces

$$
\int_{E} \alpha \varphi_{E} d s=\int_{E}\left[p_{H}\right] \cdot \tau_{E} \varphi_{E} d s=\left(p_{H}, q_{h}\right)_{L^{2}(\Omega)}
$$

with an elementwise integration by parts. Since $q_{h}=\operatorname{Curl} \varphi_{E} \in R T_{0}\left(\mathcal{T}_{h}\right)$ is an admissible test function, the discrete MFEM problem with respect to the fine mesh $\mathcal{T}_{h}$ reduces to

$$
\left(p_{h}, q_{h}\right)_{L^{2}(\Omega)}=0 .
$$

Altogether, one obtains the key identity

$$
\alpha \int_{E} \varphi_{E} d s=\left(p_{H}-p_{h}, q_{h}\right)_{L^{2}(\Omega)} .
$$

The shape regularity allows the estimates

$$
h_{E} \lesssim \int_{E} \varphi_{E} d s \quad \text { and } \quad\left\|q_{h}\right\|_{L^{2}\left(\omega_{E}\right)} \lesssim 1
$$

The foregoing key identity therefore leads to the assertion

$$
\begin{aligned}
h_{E}\|\alpha\|_{L^{2}(E)}^{2} & =h_{E}^{2}\left(\int_{E} \varphi_{E} d s\right)^{-2}\left(\alpha \int_{E} \varphi_{E} d s\right)^{2} \\
& \lesssim\left(p_{H}-p_{h}, q_{h}\right)_{L^{2}(\Omega)}^{2} \\
& \leq\left\|q_{h}\right\|_{L^{2}\left(\omega_{E}\right)}^{2}\left\|p_{h}-p_{H}\right\|_{L^{2}\left(\omega_{E}\right)}^{2} \\
& \lesssim\left\|p_{h}-p_{H}\right\|_{L^{2}\left(\omega_{E}\right)}^{2} .
\end{aligned}
$$

Lemma 4.3. There holds

$$
|\beta| \leq \frac{1}{2}\left(\left|T_{+}\right|^{-1}+\left|T_{-}\right|^{-1}\right)^{1 / 2}\left\|f-f_{\omega_{E}}\right\|_{L^{2}\left(\omega_{E}\right)} .
$$

Proof. The differences of the representation formula of Lemma 3.1 for $x \in E$ lead to

$$
\beta=\frac{1}{2}\left(f_{T_{-}}-f_{T_{+}}\right) \tau_{E} \in \mathbb{R}^{2} .
$$

Consider the piecewise constant function

$$
g(x):= \begin{cases}-\left|T_{+}\right|^{-1} & \text { for } x \in T_{+}, \\ +\left|T_{-}\right|^{-1} & \text { for } x \in T_{-}, \\ 0 & \text { for } x \notin \overline{\omega_{E}}\end{cases}
$$

and note that $\int_{\omega_{E}} g(x) d x=0$. The definition of the piecewise integral means that $f_{T_{ \pm}}:=\int_{T_{ \pm}} f(x) d x /\left|T_{ \pm}\right|$then implies the identity

$$
f_{T_{-}}-f_{T_{+}}=(g, f)_{L^{2}\left(\omega_{E}\right)} .
$$


Since $(g, 1)_{L^{2}(\Omega)}=0$ and $f_{\omega_{E}}$ is constant on $\omega_{E}$,

$$
f_{T_{-}}-f_{T_{+}}=\left(g, f-f_{\omega_{E}}\right)_{L^{2}\left(\omega_{E}\right)} .
$$

Cauchy's inequality and $\|g\|_{L^{2}\left(\omega_{E}\right)}^{2}=\left|T_{+}\right|^{-1}+\left|T_{-}\right|^{-1}$ conclude the proof:

$$
2|\beta| \leq\left(\left|T_{+}\right|^{-1}+\left|T_{-}\right|^{-1}\right)^{1 / 2}\left\|f-f_{\omega_{E}}\right\|_{L^{2}\left(\omega_{E}\right)} .
$$

The proof of Theorem 4.1 immediately follows from Lemmas 4.2 and 4.3 since $\alpha$ and $\beta \cdot(\bullet-\operatorname{mid}(E))$ are $L^{2}(E)$ orthogonal, there holds

$$
\begin{aligned}
& h_{E}\left\|\left[p_{H}\right]\right\|_{L^{2}(E)}^{2}=h_{E}\left\|\left[p_{H}\right] \cdot \tau_{E}\right\|_{L^{2}(E)}^{2} \\
& \quad=h_{E}\|\alpha\|_{L^{2}(E)}^{2}+h_{E}\|\beta \cdot(\bullet-\operatorname{mid}(E))\|_{L^{2}(E)}^{2} \\
& \quad \lesssim\left\|p_{h}-p_{H}\right\|_{L^{2}\left(\omega_{E}\right)}^{2}+h_{E}^{-1}\|(\bullet-\operatorname{mid}(E))\|_{L^{2}(E)}^{2}\left\|f-f_{\omega_{E}}\right\|_{L^{2}\left(\omega_{E}\right)}^{2}
\end{aligned}
$$

\section{Quasi-OrThogonality}

The second main argument for error reduction is a generalization of the Galerkin orthogonality in the conforming AFEM [11, 15, 16.

Theorem 5.1 (Quasi-orthogonality). There holds

$$
\begin{aligned}
\left|\left(p-p_{h}, p_{H}-p_{h}\right)_{L^{2}(\Omega)}\right| \lesssim & \left\|H\left(f_{h}-f_{H}\right)\right\|_{L^{2}(\Omega)} \\
& \times\left(\left\|p-p_{h}\right\|_{L^{2}(\Omega)}+\left\|p-p_{H}\right\|_{L^{2}(\Omega)}+\left\|H f_{H}\right\|\right)_{L^{2}(\Omega)} .
\end{aligned}
$$

Theorem 5.1 is an immediate consequence of Lemmas 5.4 and 5.5 below. Throughout the rest of this section set $p_{h}^{N}:=D_{h} u_{h}^{N}$ for the Crouzeix-Raviart FEM solution $u_{h}^{N}$ in $V_{h}^{N}$ with respect to $\mathcal{T}_{h}$.

Lemma 5.2. There holds

$$
\left(p-p_{h}^{N}, p_{H}-p_{h}\right)_{L^{2}(\Omega)}=\left(u-u_{h}^{N}, f_{H}-f_{h}\right)_{L^{2}(\Omega)} .
$$

Proof. Since $p=D u,-\operatorname{div} p_{H}=f_{H}$, and $-\operatorname{div} p_{h}=f_{h}$, the assertion follows from an elementwise integration by parts. The edge contributions vanish indeed: given any $E \in \mathcal{E}_{h}$ the resulting boundary term over $E$ reads

$$
\int_{E}\left[u-u_{h}^{N}\right]\left(p_{H}-p_{h}\right) \cdot \nu_{E} d s
$$

This is zero because $\int_{E}\left[u-u_{h}^{N}\right] d s=0$ by construction of $V_{h}^{N}$ and since $p_{H} \cdot \nu_{E}$ and $p_{h} \cdot \nu_{E}$ are continuous from both sides of $E$ and constant along $E$.

Lemma 5.3. There holds

$$
\begin{aligned}
\left|\left(u-u_{h}^{N}, f_{H}-f_{h}\right)_{L^{2}(\Omega)}\right| \lesssim \| H\left(f_{h}-f_{H}\right) & \|_{L^{2}(\Omega)} \\
& \times\left(\left\|p-p_{H}^{N}\right\|_{L^{2}(\Omega)}+\left\|p_{h}^{N}-p_{H}^{N}\right\|_{L^{2}(\Omega)}\right) .
\end{aligned}
$$

Proof. To estimate $\left(u-u_{h}^{N}, f_{H}-f_{h}\right)_{L^{2}(\Omega)}$ note that $\int_{T}\left(f_{H}-f_{h}\right) d x=0$ for any $T \in \mathcal{T}_{H}$. Hence, for some $\bar{e}_{H}^{N} \in P_{0}\left(\mathcal{T}_{H}\right)$ with

$$
\left.\bar{e}_{H}^{N}\right|_{T}:=\int_{T}\left(u(x)-u_{H}^{N}(x)\right) d x /|T|
$$


and $e_{H}^{N}:=u-u_{H}^{N}$, a Poincaré inequality on $T$ shows in total that

$$
\begin{aligned}
\left|\left(u-u_{H}^{N}, f_{H}-f_{h}\right)_{L^{2}(\Omega)}\right| & =\left|\left(e_{H}^{N}-\bar{e}_{H}^{N}, f_{H}-f_{h}\right)_{L^{2}(\Omega)}\right| \\
& \leq 1 / \pi\left\|p-p_{H}^{N}\right\|_{L^{2}(\Omega)}\left\|H\left(f_{h}-f_{H}\right)\right\|_{L^{2}(\Omega)} .
\end{aligned}
$$

The remaining term reads $\left(u_{H}^{N}-u_{h}^{N}, f_{H}-f_{h}\right)_{L^{2}(\Omega)}$ and is analyzed separately for each $T \in \mathcal{T}_{H}$. In fact, let $V_{h}^{N}(T):=\left\{\left.v_{h}\right|_{T}: v_{h} \in P_{1}\left(\left.\mathcal{T}_{h}\right|_{T}\right)\right.$ continuous at $\operatorname{mid}(E)$ for all $\left.E \in \mathcal{E}_{h}\right\}$ and note that $u_{H}^{N}-u_{h}^{N} \in V_{h}^{N}(T)$. Moreover, for any $v_{h} \in V_{h}^{N}(T)$ set

$$
\varrho_{1}\left(v_{h}\right):=\min _{w \in \mathbb{R}}\left\|v_{h}-w\right\|_{L^{2}(T)} \quad \text { and } \quad \varrho_{2}\left(v_{h}\right):=h_{T}\left\|D_{h} v_{h}\right\|_{L^{2}(T)} .
$$

This defines two semi-norms $\varrho_{1}, \varrho_{2}$ on the finite-dimensional space $V_{h}^{N}(T)$. Consequently, $\varrho_{1} \approx \varrho_{2}$. Therein, the equivalence constants are independent of $h_{T}$ according to a scaling argument (transform to a reference triangle $T_{\text {ref }}$ first and note that there exists only a finite number of possible refinements, compute the constants, and transform back). In particular, for some average $c:=\int_{T}\left(u_{H}^{N}-u_{h}^{N}\right) d x /|T|$,

$$
\begin{aligned}
\left|\left(u_{h}^{N}-u_{H}^{N}, f_{h}-f_{H}\right)_{L^{2}(T)}\right| & =\left|\left(u_{H}^{N}-u_{h}^{N}-c, f_{H}-f_{h}\right)_{L^{2}(T)}\right| \\
& \leq \varrho_{1}\left(u_{H}^{N}-u_{h}^{N}\right)\left\|f_{h}-f_{H}\right\|_{L^{2}(T)} \\
& \lesssim\left\|D_{h}\left(u_{H}^{N}-u_{h}^{N}\right)\right\|_{L^{2}(T)}\left\|h_{T}\left(f_{h}-f_{H}\right)\right\|_{L^{2}(T)} .
\end{aligned}
$$

The sum over all $T \in \mathcal{T}_{H}$ shows that

$$
\left|\left(u_{H}^{N}-u_{h}^{N}, f_{H}-f_{h}\right)_{L^{2}(\Omega)}\right| \lesssim\left\|p_{h}^{N}-p_{H}^{N}\right\|_{L^{2}(\Omega)}\left\|H\left(f_{h}-f_{H}\right)\right\|_{L^{2}(\Omega)} .
$$

Lemma 5.4. There holds

$$
\begin{aligned}
\mid\left(p-p_{h}^{N}, p_{H}\right. & \left.-p_{h}\right)_{L^{2}(\Omega)} \mid \lesssim\left\|H\left(f_{h}-f_{H}\right)\right\|_{L^{2}(\Omega)} \\
& \times\left(\left\|p-p_{h}\right\|_{L^{2}(\Omega)}+\left\|p-p_{H}\right\|_{L^{2}(\Omega)}+\left\|H f_{H}\right\|_{L^{2}(\Omega)}+\left\|h f_{h}\right\|_{L^{2}(\Omega)}\right) .
\end{aligned}
$$

Proof. The combination of Lemmas 5.2 and 5.3 readily gives

$$
\begin{aligned}
\left|\left(p-p_{h}^{N}, p_{H}-p_{h}\right)_{L^{2}(\Omega)}\right| \lesssim \| H\left(f_{h}-f_{H}\right) & \|_{L^{2}(\Omega)} \\
& \times\left(\left\|p-p_{H}^{N}\right\|_{L^{2}(\Omega)}+\left\|p_{h}^{N}-p_{H}^{N}\right\|_{L^{2}(\Omega)}\right) .
\end{aligned}
$$

An immediate consequence of Lemma 3.1 is that

$$
\begin{aligned}
\left|\left\|p-p_{H}^{N}\right\|_{L^{2}(\Omega)}-\left\|p-p_{H}\right\|_{L^{2}(\Omega)}\right|^{2} & \leq \sum_{T \in \mathcal{T}_{H}}\left|f_{T}\right|^{2}\left\|\bullet-x_{T}\right\|_{L^{2}(T)}^{2} \\
& \leq\left\|H f_{H}\right\|_{L^{2}(\Omega)}^{2} .
\end{aligned}
$$

A similar estimate also holds true with $H$ replaced by $h$. The combination of those two estimates with a triangular inequality concludes the proof of the lemma.

Lemma 5.5. There holds

$$
\left|\left(p_{h}^{N}-p_{h}, p_{H}-p_{h}\right)_{L^{2}(\Omega)}\right| \lesssim\left\|h f_{h}\right\|_{L^{2}(\Omega)}\left\|h\left(f_{h}-f_{H}\right)\right\|_{L^{2}(\Omega)} .
$$

Proof. Let $x_{H} \in P_{0}\left(\mathcal{T}_{H} ; \mathbb{R}^{2}\right)$ and $x_{h} \in P_{0}\left(\mathcal{T}_{h} ; \mathbb{R}^{2}\right)$ denote the piecewise center of inertia, e.g., $\left.x_{H}\right|_{T}:=\operatorname{mid}(T)$ for $T \in \mathcal{T}_{H}$. Then, Lemma 3.1 results in

$$
p_{H}(x)-p_{H}^{N}(x)=-\frac{1}{2} f_{H}\left(x-x_{H}\right) \quad \text { for } x \in \Omega
$$


plus a corresponding equation with $H$ replaced by $h$. Then,

$$
\begin{aligned}
\left(p_{h}^{N}-p_{h}, p_{H}-p_{h}\right)_{L^{2}(\Omega)}=\frac{1}{2} & \left(f_{h}\left(\bullet-x_{h}\right), p_{H}^{N}-p_{h}^{N}\right)_{L^{2}(\Omega)} \\
& +\frac{1}{4}\left(f_{h}\left(\bullet-x_{h}\right), f_{h}\left(\bullet-x_{h}\right)-f_{H}\left(\bullet-x_{H}\right)\right)_{L^{2}(\Omega)} .
\end{aligned}
$$

The first term on the right-hand side vanishes because $p_{H}^{N}-p_{h}^{N}$ is constant and $\int_{T}\left(x-x_{T}\right) d x=0$ for each $T \in \mathcal{T}_{h}$. The same argument shows $\left(f_{h}\left(\bullet-x_{h}\right), x_{H}-\right.$ $\left.x_{h}\right)_{L^{2}(\Omega)}=0$. There remains

$$
4\left(p_{h}^{N}-p_{h}, p_{H}-p_{h}\right)_{L^{2}(\Omega)}=\left(f_{h}\left(\bullet-x_{h}\right),\left(f_{h}-f_{H}\right)\left(\bullet-x_{h}\right)\right)_{L^{2}(\Omega)} .
$$

An elementwise Cauchy inequality in the previous identity concludes the proof.

\section{Proof of ERROR REDUCTION PROPERTY}

This section is devoted to the proof of the error reduction property (1.8) in Theorem 1.1 .

Proof. The proof starts with the reliability from Theorem 3.2 and continues with the bulk criterion (1.5), i.e.,

$$
\eta^{2}:=\sum_{E \in \mathcal{E}_{H}} h_{E}\left\|\left[p_{H}\right]\right\|_{L^{2}(E)}^{2} \lesssim \sum_{E \in \mathcal{M}} h_{E}\left\|\left[p_{H}\right]\right\|_{L^{2}(E)}^{2}
$$

for the set $\mathcal{M}$ of marked edges. This leads to

$$
\left\|p-p_{H}\right\|_{L^{2}(\Omega)}^{2} \lesssim \eta^{2}+\operatorname{osc}_{H}^{2} \lesssim \sum_{E \in \mathcal{M}} h_{E}\left\|\left[p_{H}\right]\right\|_{L^{2}(E)}^{2}+\operatorname{osc}_{H}^{2} .
$$

The discrete local efficiency of Theorem 4.1 plus the finite overlap of the edgepatches $\left(\omega_{E}: E \in \mathcal{E}_{H}\right)$ show

$$
\left\|p-p_{H}\right\|_{L^{2}(\Omega)}^{2} \lesssim \sum_{E \in \mathcal{M}}\left\|p_{h}-p_{H}\right\|_{L^{2}\left(\omega_{E}\right)}^{2}+\operatorname{osc}_{H}^{2} \leq\left\|p_{h}-p_{H}\right\|_{L^{2}(\Omega)}^{2}+\operatorname{osc}_{H}^{2}
$$

With some constant $c_{1}$, this reads

$$
\left\|p-p_{H}\right\|_{L^{2}(\Omega)}^{2} \leq c_{1}\left\|p_{h}-p_{H}\right\|_{L^{2}(\Omega)}^{2}+c_{1} \operatorname{osc}_{H}^{2} .
$$

On the other hand,

$$
\left\|p_{h}-p_{H}\right\|_{L^{2}(\Omega)}^{2}=\left\|p-p_{H}\right\|_{L^{2}(\Omega)}^{2}-\left\|p-p_{h}\right\|_{L^{2}(\Omega)}^{2}-2\left(p-p_{h}, p_{h}-p_{H}\right)_{L^{2}(\Omega)},
$$

and the last term can be bounded with the quasi-orthogonality. With some constant $c_{2}$, Theorem 5.1 leads to

$$
\begin{aligned}
\left\|p_{h}-p_{H}\right\|_{L^{2}(\Omega)}^{2} \leq & \left\|p-p_{H}\right\|_{L^{2}(\Omega)}^{2}-\left\|p-p_{h}\right\|_{L^{2}(\Omega)}^{2} \\
& \quad+c_{2}\left(\left\|p-p_{h}\right\|_{L^{2}(\Omega)}+\left\|p-p_{H}\right\|_{L^{2}(\Omega)}+\left\|H f_{H}\right\|_{L^{2}(\Omega)}\right) \operatorname{osc}_{H} .
\end{aligned}
$$

The combination with the preceding inequality plus a Young inequality yield

$$
\begin{aligned}
c_{1}\left\|p-p_{h}\right\|_{L^{2}(\Omega)}^{2} \leq & \left(c_{1}-1\right)\left\|p-p_{H}\right\|_{L^{2}(\Omega)}^{2}+c_{1} \operatorname{osc}_{H}^{2} \\
+c_{2} c_{1} & \left(\left\|p-p_{h}\right\|_{L^{2}(\Omega)}+\left\|p-p_{H}\right\|_{L^{2}(\Omega)}+\left\|H f_{H}\right\|_{L^{2}(\Omega)}\right) \operatorname{osc}_{H} \\
\leq & \frac{1}{4}\left\|p-p_{h}\right\|_{L^{2}(\Omega)}^{2}+\left(c_{1}-1 / 2\right)\left\|p-p_{H}\right\|_{L^{2}(\Omega)}^{2} \\
& +c_{4}\left(\left\|H f_{H}\right\|_{L^{2}(\Omega)}+\operatorname{osc}_{H}\right) \operatorname{osc}_{H} .
\end{aligned}
$$


This proves

$\left(c_{1}-1 / 4\right)\left\|p-p_{h}\right\|_{L^{2}(\Omega)}^{2} \leq\left(c_{1}-1 / 2\right)\left\|p-p_{H}\right\|_{L^{2}(\Omega)}^{2}+c_{4}\left(\left\|H f_{H}\right\|_{L^{2}(\Omega)}+\operatorname{osc}_{H}\right) \operatorname{osc}_{H}$, and so the theorem with

$$
\rho=\left(c_{1}-1 / 2\right) /\left(c_{1}-1 / 4\right) \text { and } C=c_{4} /\left(c_{1}-1 / 4\right) .
$$

\section{REFERENCES}

[1] Ainsworth, M. and Oden, J.T. (2000). A Posteriori Error Estimation in Finite Element Analysis. Wiley, Chichester. MR.1885308 (2003b:65001)

[2] Alonso, A. (1996). Error estimators for a mixed method. Numer. Math., 74, 4, 385-395. MR:1414415 (97g:65212)

[3] Babuska, I. and Strouboulis, T. (2001). The Finite Element Method and its Reliability. Clarendon Press, Oxford. MR1857191 (2002k:65001)

[4] Bänsch, E., Morin, P., and Nochetto, R.H. (2002). An adaptive Uzawa FEM for the Stokes problem: Convergence without the inf-sup condition. SIAM J. Numer. Anal., 40, 4, 12071229. MR 1951892 (2004e:65127)

[5] Bahriawati, C. and Carstensen, C. Three Matlab implementations of the lowest-order RaviartThomas MFEM with a posteriori error control. Newton Institute Preprint NI03069-CPD available at http://www.newton .cam.ac.uk/preprints/NI03069.pdf

[6] Bangerth, W. and Rannacher, R. (2003). Adaptive Finite Element Methods for Differential Equations. Lectures in Mathematics. ETH-Zürich. Birkhäuser, Basel. MR1960405 (2004b:65002)

[7] Binev, P., Dahmen, W., and DeVore, R. (2004). Adaptive Finite Element Methods with Convergence Rates. Numer. Math., 97, 2, 219-268. MR2050077 (2005d:65222)

[8] Brezzi, F. and Fortin, M. (1991). Mixed and hybrid finite element methods. Springer Series in Computational Mathematics, Springer-Verlag, New York, 15, x+350. MR 1115205 (92d:65187)

[9] Carstensen, C. (1997). A posteriori error estimate for the mixed finite element method. Math. Comp., 66, 218, 465-476. MR1408371 (98a:65162)

[10] Carstensen, C. and Bartels, S. (2002). Each averaging technique yields reliable a posteriori error control in FEM on unstructured grids. I. Low order conforming, nonconforming and Mixed FEM. Math. Comp., 71, 239, 945-969. MR.1898741 (2003e:65212)

[11] Dörfler, W. (1996). A convergent adaptive algorithm for Poisson's equation. SIAM J. Numer. Anal., 33, 3, 1106-1124. MR1393904 (97e:65139)

[12] Eriksson, K., Estep, D., Hansbo, P., and Johnson, C. (1995). Computational Differential Equations. Cambridge University Press, Cambridge. MR.1414897(97m:65006)

[13] Hoppe, R.H.W. and Wohlmuth, B. (1997). Adaptive multilevel techniques for mixed finite element discretizations of elliptic boundary value problems. SIAM J. Numer. Anal., 34, 16581681. MR1461801 (98e:65095)

[14] Marini, L.D. (1985). An inexpensive method for the evaluation of the solution of the lowest order Raviart-Thomas mixed method. SIAM J. Numer. Anal., 22, 3, 493-496. MR0787572 (86g:65214)

[15] Morin, P., Nochetto, R.H., and Siebert, K.G. (2000). Data oscillation and convergence of adaptive FEM. SIAM J. Numer. Anal., 38, 2, 466-488. MR.1770058 (2001g:65157)

[16] Morin, P., Nochetto, R.H., and Siebert, K.G. (2003). Local problems on stars: a posteriori error estimators, convergence, and performance. Math. Comp., 72, 243, 1067-1097. MR.1972728 (2004d:65129)

[17] Verfürth, R. (1996). A Review of A Posteriori Estimation and Adaptive Mesh-Refinement Techniques. Wiley-Teubner, New York, Stuttgart.

[18] Wohlmuth, B. and Hoppe, R.H.W. (1999). A comparison of a posteriori error estimators for mixed finite element discretizations. Math. Comp., 82, 253-279.

Department of Mathematics, Humboldt-Universität zu Berlin, D-10099 Berlin, GerMANY

Institute of Mathematics, Universität Augsburg, D-86159 Augsburg, Germany; And Department of Mathematics, University of Houston, Houston, Texas 77204-3008 